\title{
ESCARABAJOS NECRÓFAGOS (COLEOPTERA: SCARABAEIDAE Y TROGIDAE) DE LA REGIÓN CENTRAL BAJA DE VERACRUZ, MÉXICO
}

\author{
Eder F. MORA-AguILAR ${ }^{1} \&$ Enrique MONTES DE OCA ${ }^{2}$ \\ ${ }^{1}$ Instituto de Biotecnología y Ecología Aplicada, Universidad Veracruzana. Xalapa, Ver. MÉXICO. \\ 2 Autor para correspondencia. Depto. Biodiversidad y Ecología Animal, Instituto de Ecología, A.C., \\ Km. 2.5 Antigua carretera a Coatepec No. 351, Apdo 63, Congregación El Haya, Xalapa 91070, Ver., \\ MÉXICO. enrique.montesdeoca@inecol.edu.mx
}

Mora-Aguilar, E. F. y E. Montes de Oca. 2009. Escarabajos necrófagos (Coleoptera: Scarabaeidae y Trogidae) de la región central baja de Veracruz, México. Acta Zoológica Mexicana (n. s.), 25(3): 569-588.

RESUMEN. Se presenta un estudio de los escarabajos necrófagos (Scarabaeidae y Trogidae) de la región central baja de Veracruz y se compara la composición de su ensamble con otras regiones. Las colectas se realizaron durante la época de lluvias del año 2000 en sitios con remanentes derivados del bosque tropical subcaducifolio perturbado, rodeados con vegetación secundaria y cultivos de la región Apazapan-Jalcomulco establecidos entre 280 y $450 \mathrm{~m}$ snm. Se obtuvieron 2135 ejemplares pertenecientes a 3 subfamilias, 7 tribus, 11 géneros y 18 especies de Scarabaeidae y Trogidae que comprenden el $92 \%$ de las especies de escarabajos necrófagos posibles de ser encontradas en esa región. Las especies más abundantes fueron: Canthon cyanellus cyanellus, Deltochilum gibbosum sublaeve y Coprophanaeus pluto. La riqueza y abundancia fue mayor al inicio de la época de lluvias y fue disminuyendo gradualmente. Se encontró mayor abundancia en Apazapan que en Jalcomulco. Los escarabajos cavadores superan a los rodadores en riqueza específica en proporción de 3:1en tanto que la proporción en abundancia fue inversa. La actividad de especies nocturnas sobrepasa la de diurnas en la misma proporción. Esta región presenta hasta el 63\% de similitud específica y alrededor del $80 \%$ de similitud genérica con la región costera de Veracruz, valores comparables con el $56 \%$ y el $90 \%$ de similitudes respectivas con la parte baja de la Reserva de la Biósfera El Cielo, Tamaulipas.

Palabras clave: escarabajos necrófagos, región central baja, Veracruz, bosque tropical subcaducifolio.

Mora-Aguilar, E. F., \& E. Montes de Oca. 2009. Necrophagous beteles (Coleoptera: Scarabaeidae and Trogidae) from lowland central Veracruz, Mexico. Acta Zoológica Mexicana (n. s.), 25(3): 569-588. ABSTRACT. The necrophagous beetles (Scarabaeidae and Trogidae) occurring in the lowland of central Veracruz are studied and the assemblage composition is compared with those found in other regions. Specimens were collected during the rainy season of 2000 in the disturbed semi deciduous seasonal forest remnants associated with secondary vegetation and crop fields of Apazapan-Jalcomulco region (280-450 m of altitude). We recorded 2135 specimens belonging to the Scarabaeidae and Trogidae families represented in three subfamilies, seven tribes, 11 genera, and 18 species which represent the $92 \%$ of the all necrophagous beetle species possible to be found in this region. Canthon cyanellus cyanellus, Deltochilum gibbosum sublaeve y Coprophanaeus pluto were the most abundant 
species on the samples. Richness and abundance were higher at the beginning of the rainy season followed by a gradual decrease as the season went by. Beetle abundance was higher in Apazapan than in Jalcomulco. Burrower beetle species richness exceeded the roller species in a 3:1 ratio but the abundance ratio was reversed. Daily beetle activity of nocturnal species is higher than diurnal species. This region showed $63 \%$ of specific similarity and around $80 \%$ of genus similarity with the coastal region of Veracruz, and is comparable with $56 \%$ and $90 \%$ of the respective similarities with the lowland region of "El Cielo" Biosphere Reserve in Tamaulipas.

Key words: necrophagous beetles, lowland central Veracruz, tropical semi deciduous forest.

\section{INTRODUCCIÓN}

En México la entomofauna necrófila comenzó a estudiarse sistemáticamente desde fines de los años setentas y principios de los ochentas. Los trabajos realizados en buena parte del país, en distintas regiones y tipos de vegetación han generado un buen nivel de conocimiento de aspectos ecológicos, biogeográficos y taxonómicos de uno de sus principales componentes, los escarabajos copro-necrófagos (ver síntesis en Morón 1996, 2003). Por el auge de aquellos estudios faunísticos se facilitó el reconocimiento de este grupo de coleópteros como buenos objetos de estudio para la interpretación y comprensión de la diversidad biológica. La necesidad de evaluar los efectos antropogénicos en sitios otrora provistos con cubierta vegetal original o con poco disturbio ha promovido el uso de este grupo de insectos como indicadores de cambios en su diversidad al comparar con ambientes similares de otras regiones (Halffter et al. 1992).

En este trabajo se analiza el ensamble de escarabajos necrófagos (Coleoptera: Scarabaeidae y Trogidae) de la región central baja del estado de Veracruz. En este lugar se ha venido desarrollando con éxito el turismo ecológico y la práctica de deportes extremos como actividad económica alternativa a la agricultura. Su intensificación en los últimos 15 años ha incrementado la presión sobre los reductos de vegetación primaria y secundaria madura a través de mayor demanda de recursos forestales y cambios de uso de suelo para satisfacer las necesidades de visitantes, nuevos colonizadores y de los procesos de urbanización. La permanencia de algunas especies de escarabajos necrófagos podría verse afectada ante la reducción de sitios con ambientes naturales y derivados, por lo que aquí se presente una comparación de las especies de esta región con otras de ambientes similares.

\section{MATERIAL Y MÉTODOS}

Área de estudio. La región central baja de Veracruz se encuentra sobre las últimas estribaciones orientales del Cofre de Perote. Comprende dos municipios contiguos Apazapan y Jalcomulco (Fig. 1). El primero es recorrido por la barranca del mismo nombre; entre los $19^{\circ} 19^{\prime} \mathrm{N}$ y $96^{\circ} 43^{\prime} \mathrm{O}$, con una altura media de 300 metros sobre el nivel del mar presentando una orografía irregular. Su clima es cálido subhúmedo, con una temperatura media anual de $25.5^{\circ} \mathrm{C}$, con lluvias abundantes en el verano y 


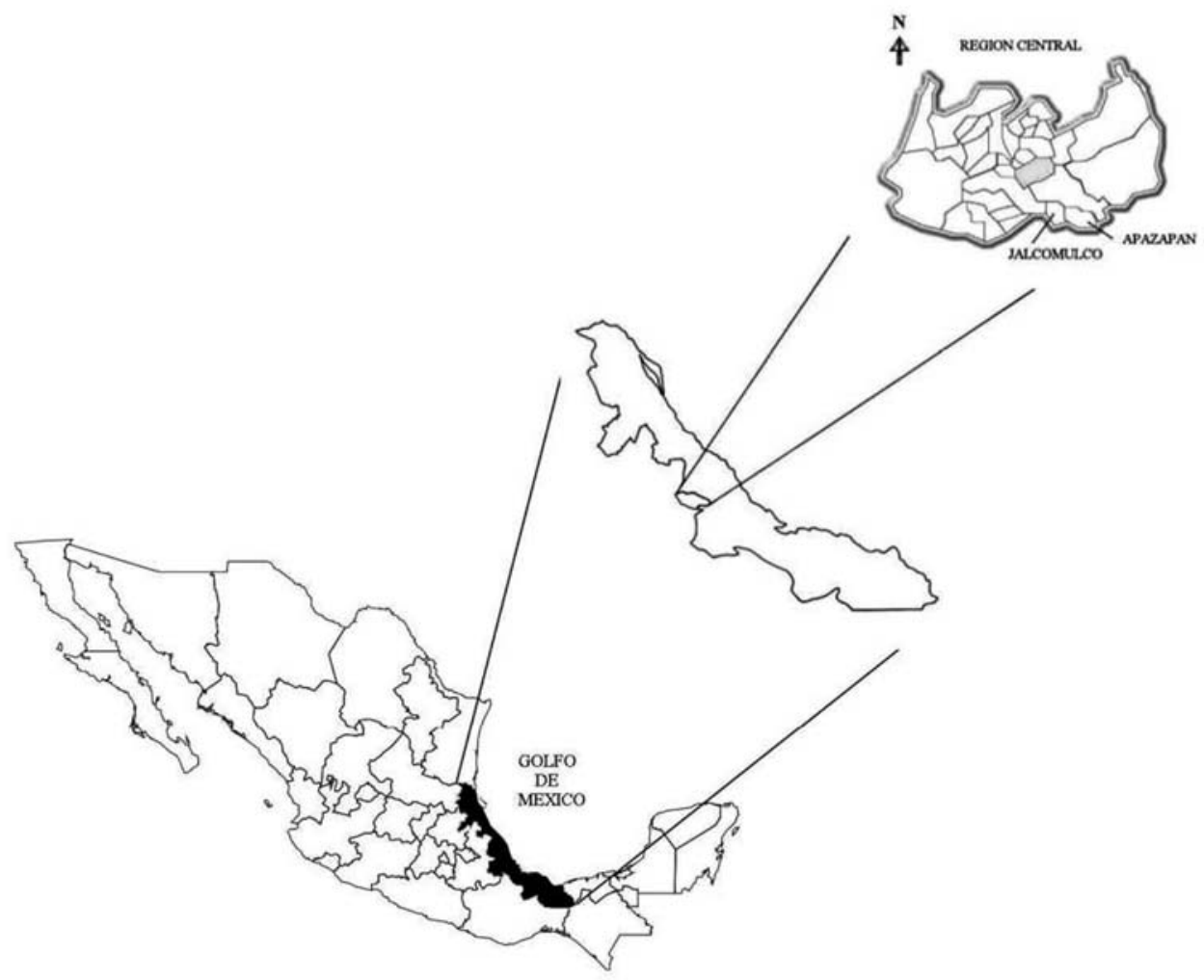

Figura 1. Ubicación del área de estudio en la región central de Veracruz.

a principios del otoño, y lloviznas en invierno por la influencia de vientos del norte. Su precipitación pluvial media anual es de $1,250 \mathrm{~mm}$. Se encuentran suelos de tipos feozem (capa oscura, suave y rica en materia orgánica y nutrientes) y rendsina (capa superficial de materia orgánica que descansa sobre roca caliza) en los que coexisten el bosque tropical subcaducifolio con poblaciones de Cecropia peltata L. (guarumbo), Cecropia obtusifolia Bertol. (chancarro), Heliocarpus pallidus Rose (jonote), Heliocarpus mexicanus (Turcz.) (guanacaxtle), Enterolobium cyclocarpum (Jacq.) Griseb. y encinares (Quercus peduncularis Née, Q. polymorpha Schltdl. \& Cham., $Q$. oleoides Schltdl.). Se desarrolla la agricultura, donde maíz, frijol, calabaza, plátano, cítricos y mango son los cultivos comunes, y en mucho menor escala la ganadería, principalmente bovina. Limitando al este con Apazapan, el municipio de Jalcomulco se ubica entre los $19^{\circ} 20^{\prime} \mathrm{N}$ y $96^{\circ} 46^{\prime} \mathrm{O}$, a una altura media de 350 metros sobre el nivel del mar, encontrándose accidentes depresivos que forman las barrancas de Tuzamapan y Jalcomulco. Su clima es también cálido 
subhúmedo con una temperatura promedio anual de $24^{\circ} \mathrm{C}$, lluvias abundantes en verano y principios de otoño. Su precipitación pluvial media anual es de 1,125 mm. Presenta suelos de tipo feozem, rendsina y luvisol (acumulación de arcilla en el subsuelo) donde se desarrollan el bosque tropical subcaducifolio y caducifolio con Ipomoea wolcottiana Rose (palo bobo), Lysiloma divaricatum (Jacq.) J.F. McBr. (rajador), Eysenhardtia polystachia (Ortega) Sarg. (vara dulce), Brosimum alicastrum (L.) Sarg. (chaca), Guazuma ulmifolia Lam. (guacima), Piscidia piscipula (L.) Sarg. (espino chijal) y Mimosa sp. (raspillo). Se practica en mayor porcentaje la agricultura (maíz, caña de azúcar, café y mango) y en menor proporción la cría de ganado. Esta región central baja es regada por el río de Los Pescados o de La Antigua, y presenta una marcada estacionalidad (época de lluvias: Mayo-Octubre; época de secas: Noviembre-Abril, Fig. 2).

Sitios de muestreo. Se escogieron tres sitios de muestreo: dos en Apazapan (APAZ1, APAZ2) y uno en Jalcomulco (JALC), en función de la accesibilidad y posibilidades de muestreo. Cada sitio representa un hábitat con cobertura vegetal arbórea (> $8 \mathrm{~m}$ altura) con una extensión aproximada de 4 a 5 ha (APAZ1.plantación de mango, $19^{\circ} 19.18^{\prime} \mathrm{N} / 96^{\circ} 42.39^{\prime}$ O; APAZ2.- fragmento de bosque,

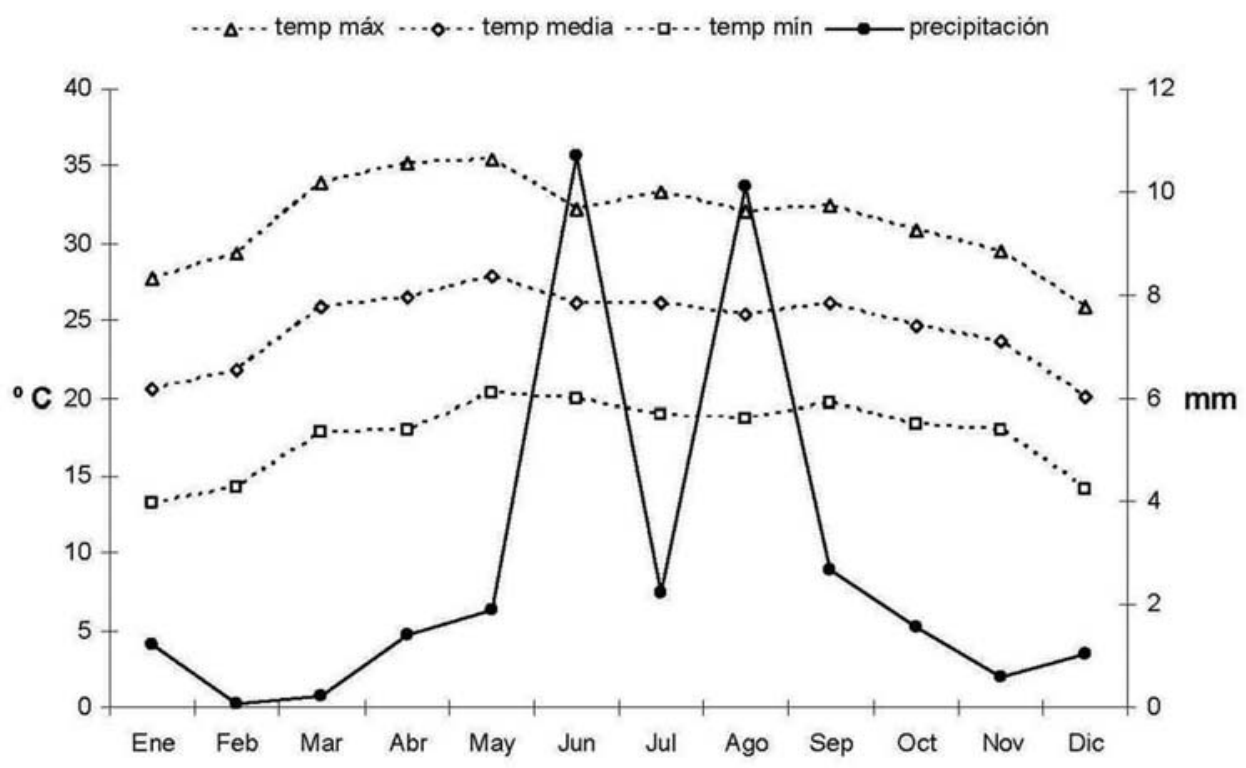

Figura 2. Diagrama ombrotérmico de la zona de estudio elaborado con datos del 2000 proporcionados por la CONAGUA. 
$19^{\circ} 19.43^{\prime} \mathrm{N} / 96^{\circ} 44.26^{\prime} \mathrm{O}$ y JALC.- café de sombra con árboles nativos y plátano, $\left.19^{\circ} 19.86^{\prime} \mathrm{N} / 96^{\circ} 45.77^{\prime} \mathrm{O}\right)$. No fue posible encontrar fragmentos de bosque adecuados para el estudio ya que los remanentes se encuentran inaccesibles en pendientes pronunciadas de las barrancas donde el suelo es rocoso. Las plantaciones elegidas no son manejadas intensivamente y tienen más de 20 años semejando la estructura de un bosque. Los tres sitios colindaban con área de cultivo (de maíz intermezclado con frijol y calabaza) y área abierta (campo de cultivo abandonado sujeto a pastoreo intermitente). La distancia entre APAZ1 Y APAZ2 es cuando menos de un kilómetro y de varios kilómetros entre éstos y JALC.

Recolecta. Los escarabajos necrófagos se colectaron en cada sitio mediante trampas tipo NTP-80 (Morón y Terrón 1984) cebadas con trozos de calamar. La revisión de trampas y renovación de los cebos se realizó cada 30 días durante cinco meses a partir del inicio de la época de lluvias, iniciando a fines de Mayo y finalizando en Noviembre de 2000. Este período abarca la época de mayor actividad de los escarabajos. En la figura 2 se presentan las variables climáticas durante el período de muestreo.

El material extraído de las trampas fue lavado y mantenido en alcohol $70 \%$ para su posterior determinación. Se montaron en alfiler especímenes de las diferentes especies encontradas y se conformó una colección de referencia. Una vez identificados mediante claves dicotómicas, el resto de los especímenes se cuantificó y colocó en camas de algodón para su secado y preservación. Todo el material (montado y seco) se encuentra temporalmente bajo resguardo de uno de los autores (EMO) para su posterior inclusión en la Colección Entomológica IEXA.

Análisis. Se determinó la diversidad empleando los estimadores Mao Tau, ACE Mean, ICE Mean, Chao 2 Mean, Jacknife1 Mean y MMMean del programa EstimateSWin700 (Colwell 2005). Se elaboró la curva de acumulación de especies (Soberón y Llorente 1993, León-Cortés et al. 1998 y Jiménez-Valverde \& Hortal 2003) para analizar la riqueza específica de las muestras y evaluar la efectividad del muestreo como inventario de la fauna necrófaga de la región utilizando la ecuación asintótica de Clench, $S(y)=a x /(1+b x)$ y ajustando la regresión no lineal mediante Statistica (StatSoft, 1999) donde $\mathrm{R}^{2}$ representa una medida descriptiva de la proporción de la varianza explicada por la función, sus valores van de 0 (los datos no se ajustan a la función) a 1 (los datos se ajustan totalmente a la función). Este modelo permite predecir el número de especies al cual una curva de acumulación llega a una asíntota (calculado como la relación $a / b$ ). También es posible calcular: a) la calidad del inventario mediante el cálculo de la pendiente al final de la curva, $\left.a /(1+b n)^{2} ; \mathrm{b}\right)$ la proporción de la fauna registrada, $n_{q}=q /(b(1-q))$ donde $q=S_{n} /(a / b)$ y c) el esfuerzo requerido para registrar una determinada proporción de la fauna $\left(n_{q}\right)$, por ejemplo 95\% de las especies. Ya que el orden en que las muestras se añaden al total puede afectar la forma de la curva, se aleatorizó el orden de muestreo (Colwell y 
Coddington 1994) utilizando el procedimiento para ello en el programa EstimateSWin700 (Colwell 2005).

Una vez verificados los supuestos de homocedasticidad y normalidad, se realizaron análisis de varianza para cada variable dependiente (número de especies y número de individuos) para analizar los efectos no interactivos de primer orden de las dos variables categóricas independientes o factores (sitio y período de muestreo). Para el caso de la abundancia el número de individuos se transformó a logaritmos en base 10. En los casos de efectos significativos se realizaron las pruebas post-hoc correspondientes.

Se realizaron comparaciones de los resultados obtenidos en esta región con las de otras del estado de Veracruz y del país en donde se presentan condiciones, ambientes y tipos de vegetación similares o equivalentes (bosque tropical subcaducifolio sensu Rzedowski 1986) que se encuentran publicadas. Se utilizó el índice cualitativo de similitud/disimilitud de Sörensen. Se elaboró una clave dicotómica para ayudar a la determinación de las especies encontradas en esta región central baja de Veracruz (Apéndice).

Clave dicotómica. Se elaboró modificando claves y revisiones y compilaciones existentes (Arnaud, 2002; Delgado \& Kohlman, 2007; Deloya, 2005; Deloya et al. 2007; Edmonds, 1994; Howden, 1966; Howden \& Cartwright, 1963; Morón 2003; Morón, 1979; Jessop, 1985; Kohlmann \& Solis, 2001; Rivera-Cervantes \& Halffter, 1999) para determinar las especies necrófagas propias del bosque tropical caducifolio del centro de Veracruz facilitando así la determinación de las especies de la región y evitar confusiones con especies afines o que se sobreponen en sus distribuciones.

\section{RESULTADOS}

Se colectaron 2135 ejemplares adultos pertenecientes a las familias Scarabaeidae y Trogidae que representan a 18 especies agrupadas en 11 géneros, 7 tribus y 3 subfamilias (Cuadro 1). Los Scarabaeidae conformaron el $97.5 \%$ de los individuos y el $89 \%$ de las especies.

Efectividad del inventario. De acuerdo a algunos estimadores de riqueza específica se podrían esperar de dos a tres especies más a las obtenidas en este muestreo (18 \pm 3.45 (Mao Tau 95\% IC); $18 \pm 1.76$ (Mao Tau SD), ACE Mean: 21.97, ICE Mean: 21.45, Chao 2 Mean: 20.22, Jacknife1 Mean: 20.22, MMMean: 18.88). El modelo asintótico de Clench explicó el $97.4 \%$ de la proporción de la varianza en la curva de acumulación de especies de los tres sitios y predice que el número total de especies a encontrarse es de 19.6 ( $a=0.0736$ y $b=0.0037$ ) (Figura 3). La proporción de la fauna registrada fue de $91.9 \%$. En caso de querer aumentar a $95 \%$ la proporción de fauna a registrar tendríamos que colectar 5053 individuos, esto es 2918 individuos más para aumentar dos especies, lo cual implicaría que para obtener un inventario completo habría que triplicar el esfuerzo a 15 muestreos. 
Cuadro 1. Nombre de las especies de coleópteros Scarabaeidae y Trogidae capturadas por sitio de muestreo con trampas NTP-80 durante los meses de Junio a Noviembre de 2000. APAZ= Apazapan, $\mathrm{JALC}=$ Jalcomulco.

\section{SCARABAEIDAE}

APAZ1 APAZ2 JALC

Scarabaeinae

Scarabaeini

Deltochilum (Deltohyboma) scabriusculum scabriusculum Bates, 1887

Deltochilum (Hybomidium) gibbosum sublaeve Bates, 1887

Canthon (Glaphyrocanthon) moroni Rivera-Cervantes \& Halffter, 1999 *

Canthon (Glaphyrocanthon) leechi (Martinez, Halffter \& Halffter, 1964)

Canthon (Glaphyrocanthon) zuninoi Rivera-Cervantes \& Halffter, 1999 *

Canthon (Canthon) cyanellus cyanellus LeConte, 1859

Sisyphus mexicanus Harold, 1863

Eurysternini

Eurysternus mexicanus Harold, 1869

$\mathrm{X}$

Coprini

Dichotomius amplicollis (Harold, 1869)

Uroxys deavilai Delgado \& Kohlmann 2007

$\begin{array}{lll}X & X & X \\ X & X & -\end{array}$

Phanaeini

Coprophanaeus (Coprophanaeus) telamon corythus (Harold, 1863)

Coprophanaeus (Coprophanaeus) pluto (Harold, 1863)

Phanaeus (Phanaeus) tridens Laporte de Castelnau, 1840

Phanaeus (Notiophanaeus) endymion Harold, 1863

$\begin{array}{ccc}- & - & \text { X } \\ X & X & \text { X } \\ X & X & \text { X } \\ \text { X } & - & \text { X }\end{array}$

Onthophagini

Onthophagus (Onthophagus) batesi Howden \& Cartwright, 1963

Onthophagus (Onthophagus) höpfneri Harold 1869

Onthophagus (Onthophagus) schaefferi Howden \& Cartwright 1863

$\begin{array}{ccc}\text { X } & \text { X } & \text { X } \\ \text { X } & \text { X } & \text { X } \\ - & - & - \\ \text { X } & \text { X } & \text { X } \\ - & - & - \\ \text { X } & \text { X } & \text { X } \\ - & \text { X } & -\end{array}$

Aphodiinae

Eupariini

Ataenius (Ataenius) cribrithorax Bates, 1887

$\mathrm{X}$

\section{TROGIDAE}

\section{Troginae}

Omorgus fuliginosus Robinson, 1941

Omorgus suberosus Fabricius, 1775

$\begin{array}{lll}X & X & X \\ X & - & X\end{array}$

\footnotetext{
* Especies no registradas en este trabajo pero que pueden encontrarse en esa localidad según Rivera-Cervantes \& Halffter 1999.
} 


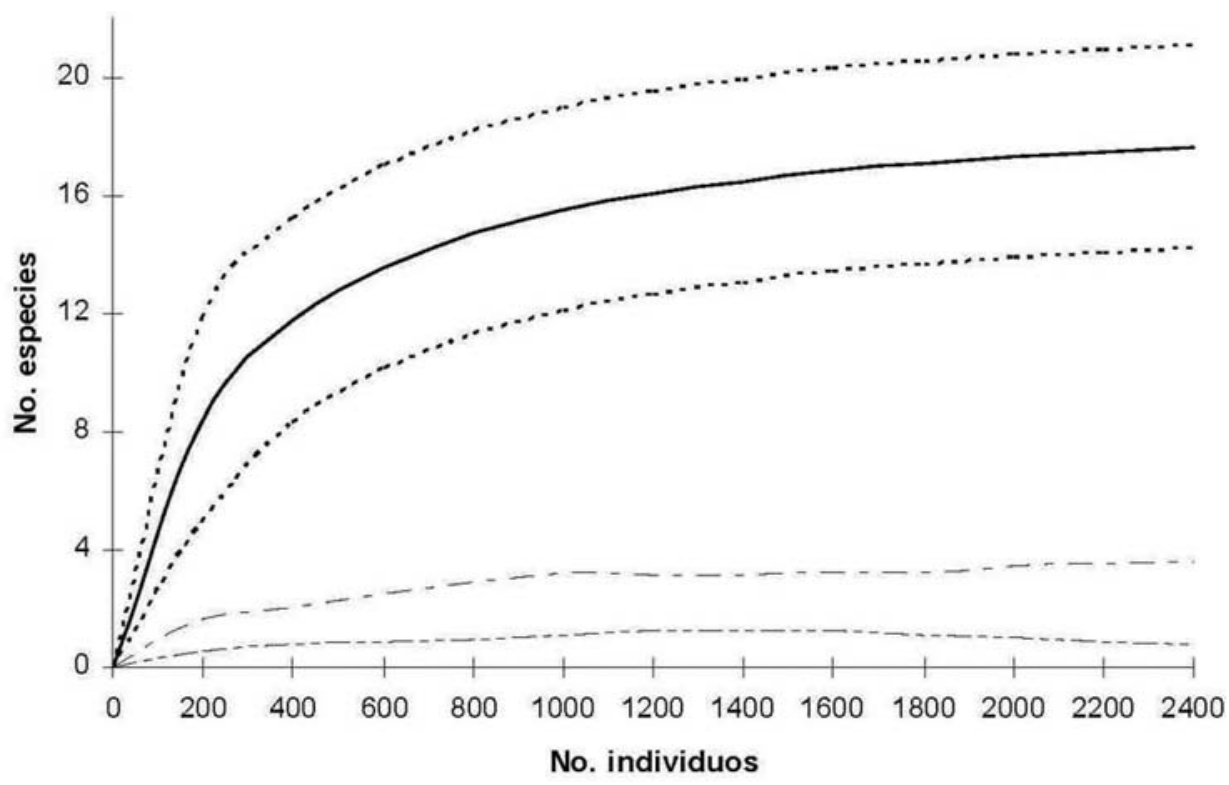

Figura 3. Curva de acumulación de especies de escarabajos colectados en zonas arboladas de la región central baja del centro de Veracruz. Líneas punteadas representan el intervalo a 95\% de confianza (estimador Mao Tau). Las dos líneas interrumpidas de la parte inferior indican los "singletons" y "doubletons" respectivamente (ver texto).

Los "singletons" se refieren a las especies únicas con un solo espécimen que se colectaron en una sola ocasión y en un solo sitio de muestreo (un sola muestra) fueron Onthophagus batesi Howden \& Cartwright, 1963, O. höpfneri Harold, 1869, Eurysternus mexicanus Harold, 1869 (APAZ1) y Ataenius cribrithorax Bates, 1887 (JALC). Los "doubletons", como el caso de Omorgus suberosus Fabricius, 1775 se refiere a especies encontradas en dos muestras (APAZ1 y JALC).

Sisyphus mexicanus Harold, 1863 y Coprophanaeus telamon corythus (Harold, 1863) fueron colectados en varias ocasiones pero sólo en un sitio, APAZ2 y JALC respectivamente, mientras que Uroxys deavilai Delgado \& Kohlmann, 2007 se encontró tan sólo en el primer período de muestreo en los dos sitios de Apazapan. Por su parte Phanaeus endymion Harold, 1863 se encontró en un solo período en un sitio (APAZ1) y en varios períodos en otro (JALC). Las nueve especies restantes se encontraron en los tres sitios, siendo las más importantes por su abundancia, Canthon cyanellus cyanellus LeConte, 1859 (31.8\%), Deltochilum gibossum subleave Bates, 1887 (28.4\%), Coprophanaeus pluto (Harold, 1863) (10.6\%), Canthon (Glaphyrocanthon) leechi (Martinez, Halffter \& Halffter, 1964) (9.4\%), Onthophagus schaefferi Howden \& Cartwright, 1963 (8.5\%) y Dichotomius 
amplicollis (Harold, 1869) (5.6\%) seguido por Omorgus fuliginosus Robinson, 1941 (2.4\%) y finalmente por Deltochilum scabriusculum scabriusculum Bates, 1887 (0.7\%) y Phanaeus tridens Laporte, 1840 (0.5\%) (Cuadro 2). Sin embargo la dominancia de la mayoría de éstas, establecida por su abundancia relativa en cada sitio, varía en mayor o menor grado en los tres sitios muestreados (Figura 4).

La complementariedad entre los sitios de muestreo fue relativamente baja y muy similar entre sí con un promedio de $37.6 \%$ (Figura 4). De aquí que no se detectara diferencia estadísticamente significativa en el número de especies entre los tres sitios muestreados $\left(\mathrm{F}_{2,8}=1.000, \mathrm{p}=0.4096\right)$. No obstante sí hubo una variación significativa en el número de especies a lo largo de los períodos de muestreo $\left(\mathrm{F}_{4,8}=11.140, \mathrm{p}<\right.$ 0.01) con mayor número de especies al inicio de la época de lluvias (Junio y Julio) y una disminución gradual hacia el último período de colecta (Octubre-Noviembre). Se encontró mayor abundancia en Apazapan que en Jalcomulco $\left(\mathrm{F}_{2,8}=22.983, \mathrm{p}<0.001\right)$ y una disminución gradual en el número de individuos a partir de junio $\left(\mathrm{F}_{4,8}=26.188\right.$, $\mathrm{p}<0.0001)$.

Cuadro 2. Abundancia relativa de los Scarabaeidae y Trogidae necrófagos capturados con trampas tipo NTP-80 en Apazapan y Jalcomulco, Veracruz durante la época de lluvias.

\begin{tabular}{lcccccc}
\hline Especies & Jun & Jul & Ago & Sep & Oct-Nov & Totales \\
\hline D. s. scabriusculum & 9 & 4 & 1 & - & - & 14 \\
D. g. subleave & 241 & 119 & 117 & 98 & 32 & 607 \\
C. leechi & 15 & 76 & 45 & 41 & 24 & 201 \\
C. c. cyanellus & 334 & 171 & 113 & 49 & 11 & 678 \\
S. mexicanus & - & 1 & 1 & 1 & 1 & 4 \\
E. mexicanus & 1 & - & - & - & - & 1 \\
D. amplicollis & 86 & 29 & 4 & 1 & - & 120 \\
U. deavilai & 4 & - & - & - & - & 4 \\
C. t. corythus & 2 & 1 & - & 2 & - & 5 \\
C. pluto & 85 & 56 & 37 & 35 & 14 & 227 \\
P. tridens & 8 & 3 & - & - & - & 11 \\
P. endymion & 20 & 2 & 2 & 1 & - & 25 \\
O. batesi & - & - & 1 & - & - & 1 \\
O. höpfneri & 1 & - & - & - & - & 1 \\
O. schaefferi & 42 & 19 & 39 & 48 & 34 & 182 \\
A. cribritorax & - & - & - & 1 & - & 1 \\
O. fuliginosus & 22 & 16 & 4 & 8 & 1 & 51 \\
O suberosus & 1 & 1 & - & - & - & 2 \\
Totales por mes & 871 & 498 & 364 & 285 & 117 & 2135 \\
\hline No. de especies & 15 & 13 & 11 & 11 & 7 & 18 \\
\hline & & & & & & \\
\hline
\end{tabular}




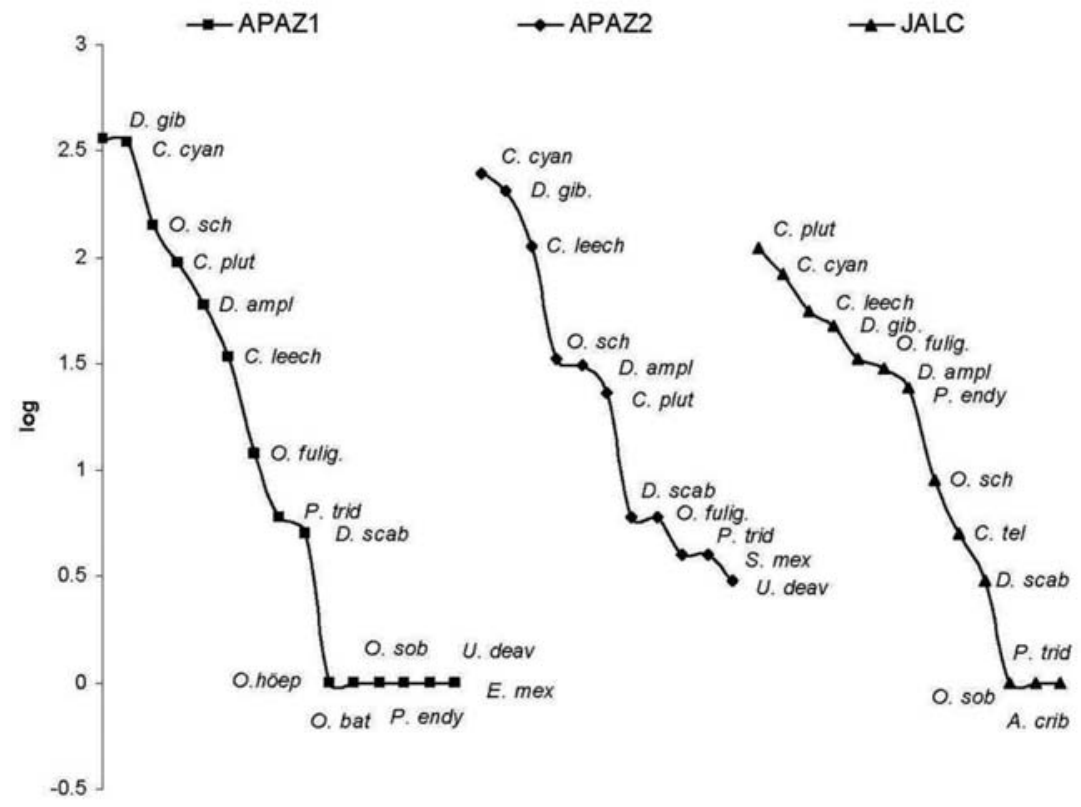

Figura 4. Gráficas de dominancia para los escarabajos colectados con NTP80 en tres sitios de la región central baja de Veracruz. La complementariedad (Colwell y Coddington 1994) entre sitios fue:

$$
\mathrm{C}_{\mathrm{APAZ1}-\mathrm{APAZ2}}=0.375, \mathrm{C}_{\mathrm{APAZ1}-\mathrm{JALC}}=0.353 \mathrm{y} \mathrm{C}_{\mathrm{APAZ2}-\mathrm{JALC}}=0.400
$$

Hábitos. Las especies consideradas como necrófagas especialistas, dada la abundancia con que fueron colectadas son Canthon cyanellus, Deltochilum gibossum, Coprophanaeus pluto. En tanto las especies con abundancia intermedia a baja son consideradas copro-necrófagas o coprófagas no estrictas, esto es, especies más generalistas que son atraídas por el cebo utilizado en esta ocasión (Cuadro 3). Aquellas especies con apenas uno o dos individuos no son consideradas precisamente necrófagas sino que tienen una afinidad mayor hacia la coprofagia o saprofagia y su captura es incidental.

Del total de los ejemplares colectados, la abundancia de las especies con hábitos nocturnos fue ligeramente superior a las especies diurnas (58.08\% y 41.92). Hubo una proporción de 3 a 1 entre el total de especies cavadoras y rodadoras, compuestas por $72.22 \%$ nocturnas y $27.78 \%$ diurnas. Se observa el predominio en abundancia de los escarabajos rodadores (70.49\%) ante los cavadores (29.51\%). Los rodadores diurnos son poco mas abundantes que los nocturnos, $58.67 \%$ y $41.33 \%$ respectivamente. Entre las especies cavadoras la abundancia de las especies nocturnas (98.1\%) es superior a las diurnas (1.9\%) (Cuadro 3). 
Cuadro 3. Caracterización bioecológica de las especies colectadas en este trabajo.

$\mathrm{N}=$ Necrófaga, $\mathrm{C}=$ Coprófaga, $\mathrm{T}=$ Telionecrófaga, $\mathrm{S}=$ Saprófaga; Noc= Nocturna, Diu= Diurna;

$\mathrm{Ca}=$ Cavador, $\mathrm{Ro}=$ Rodador; $(\mathrm{i})=$ incidental

Especies

Hábitos de Alimentación

Actividad

\section{Este trabajo Literatura}

\begin{tabular}{|c|c|c|c|c|}
\hline D. s. scabriusculum & $\mathrm{C}-\mathrm{N}$ & $\mathrm{N}-\mathrm{C}$ & Ro & Noc \\
\hline D. g. sublave & $\mathrm{N}$ & $\mathrm{N}-\mathrm{C}$ & Ro & Noc \\
\hline C. leechi & $\mathrm{C}-\mathrm{N}$ & $\mathrm{C}-\mathrm{N}$ & Ro & Diur \\
\hline C. c. cyanellus & $\mathrm{N}$ & $\mathrm{N}$ & Ro & Diur \\
\hline S. mexicanus & $\mathrm{C}-\mathrm{N}$ & $\mathrm{C}$ & Ro & Diur \\
\hline E. mexicanus & C-S (i) & $\mathrm{C}-\mathrm{N}$ & $\mathrm{Ca}$ & Noc \\
\hline D. amplicollis & $\mathrm{C}-\mathrm{N}$ & $\mathrm{C}-\mathrm{N}$ & $\mathrm{Ca}$ & Noc \\
\hline U. deavilai & $\mathrm{C}-\mathrm{N}$ & $\mathrm{C}-\mathrm{N}$ & $\mathrm{Ca}$ & Noc \\
\hline C. t. corythus & $\mathrm{C}-\mathrm{N}$ & $\mathrm{N}-\mathrm{C}$ & $\mathrm{Ca}$ & Noc \\
\hline C. pluto & $\mathrm{N}$ & $\mathrm{N}-\mathrm{C}$ & $\mathrm{Ca}$ & Noc \\
\hline P. tridens & $\mathrm{C}-\mathrm{N}$ & $\mathrm{C}$ & $\mathrm{Ca}$ & Diur \\
\hline P. endymion & $\mathrm{C}-\mathrm{N}$ & $\mathrm{C}-\mathrm{N}$ & $\mathrm{Ca}$ & Noc \\
\hline O. batesi & C-S (i) & $\mathrm{C}$ & $\mathrm{Ca}$ & Noc \\
\hline O. höpfneri & C-S (i) & $\mathrm{C}$ & $\mathrm{Ca}$ & Diur \\
\hline O. schaefferi & $\mathrm{C}-\mathrm{N}$ & $\mathrm{C}$ & $\mathrm{Ca}$ & Noc \\
\hline A. cribrithorax & C-S (i) & $\mathrm{C}$ & $\mathrm{Ca}$ & Noc \\
\hline O. fuliginosus & $\mathrm{C}-\mathrm{N}$ & $\mathrm{T}-\mathrm{C}$ & $\mathrm{Ca}$ & Noc \\
\hline O. suberosus & C-S (i) & $\mathrm{T}-\mathrm{C}$ & $\mathrm{Ca}$ & Noc \\
\hline
\end{tabular}

Relaciones faunísticas. Al comparar los componentes de esta fauna de Apazapan y Jalcomulco con otras faunas con vegetación tropical similar o equivalente por medio del índice de similitud de Sörensen, observamos a nivel genérico valores altos, mostrando porcentajes mayores al $60 \%$. La región con mayor afinidad es la región de El Cielo (90\%) y Palma Sola (81.8\%). En segundo término algunas regiones presentaron porcentajes intermedios como, Laguna Verde (72.7\%), Acahuizotla (72.7\%), Los Tuxtlas (72\%) y Puerto Ángel (70\%). Por último, las regiones con porcentajes menores se observaron en Chamela (63.6\%), Jojutla (63.1\%).

A nivel específico los resultados fueron diferentes, donde la afinidad más alta se encontró con Palma Sola (62.9\%), en segundo término la Reserva de la Biósfera El Cielo (56.4\%) y Laguna Verde (55\%). Con las regiones restantes, (Jojutla, Chamela, Los Tuxtlas, Acahuizotla y Puerto Ángel) se obtuvo un porcentaje bajo $(<35 \%)$ a pesar de presentar un tipo de vegetación similar, bosque tropical caducifolio y subcaducifolio, con excepción del bosque tropical perennifolio de Los Tuxtlas (Cuadro 4). 
Mora-Aguilar y Montes de Oca: Escarabajos necrófagos del centro de Veracruz

Cuadro 4. Índice de Similitud de Sörensen (\%) obtenidos al comparar riquezas de Scarabaeidae de diferentes localidades mexicanas con Apazapan-Jalcomulco. Btc $=$ Bosque tropical caducifolio, $\mathrm{Btp}=$ Bosque tropical perennifolio, $\mathrm{Btsc}=\mathrm{B}$ osque tropical subcaducifolio, $\mathrm{P}=\mathrm{Pastizal}$ inducido.

\begin{tabular}{lccccccc}
\hline Localidades & \multicolumn{2}{c}{ Riqueza de } & \multicolumn{2}{c}{ Similitud (\%) } & & $\begin{array}{c}\text { Vegetación } \\
\text { (sensu Rzedowski, 1973) }\end{array}$ & $\begin{array}{c}\text { Altitud } \\
\text { (msnm) }\end{array}$ \\
\cline { 2 - 5 } & Especies & Géneros & Específica & Genérica & & Btsc, P & $42-370$ \\
\hline Palma Sola, Ver. 1 & 17 & 11 & 62.9 & 81.8 & & Btc, P & 150 \\
Lag. Verde, Ver. 2 & 22 & 11 & 55 & 72.7 & & Btp, P & $150-530$ \\
Los Tuxtlas, Ver. 3 & 24 & 14 & 33.3 & 72 & & Btc & 100 \\
Pto. Ángel, Oax. 4 & 12 & 9 & 20 & 70 & & Btc & 100 \\
Chamela, Jal. 5 & 16 & 11 & 29.4 & 63.6 & & Btc & $800-1550$ \\
Jojutla, Mor. 6 & 14 & 8 & 31.3 & 63.1 & & Btsc & 800 \\
Acahuizotla, Gro.7 & 23 & 11 & 34.2 & 72.7 & & Btsc & $200-800$ \\
El Cielo, Tamps. 8 & 21 & 9 & 56.4 & 90 & & & \\
\hline
\end{tabular}

Fuentes: ${ }^{1}$ Montes de Oca \& Halffter 1995, 2Arellano \& Halffter, 2003, 3, Morón 1979, 4Deloya \& Morón 1998, 5Morón et al., 1989, 6Deloya \& Morón 1994, 7Delgado 1989, 8Delgado \& Montes de Oca 2005.

\section{DISCUSIÓN}

El muestreo realizado en este trabajo puede considerarse lo suficientemente exhaustivo y confiable ya que se ha reconocido $91.9 \%$ de la fauna de escarabajos que es posible encontrar. El esfuerzo requerido para completar el inventario no es justificable en sentido práctico y mucho menos de sacrificio inútil de varios cientos de especímenes más. En adición, se registró el 3.7\%, $11.1 \%$ y $9.6 \%$ de las especies copro-necrófagas reportadas para México, Veracruz y en los Bosques Tropicales Caducifolios del país, respectivamente.

Las 18 especies de Scarabaeidae y Trogidae obtenidas con NTP-80 en Apazapan y Jalcomulco son comparables a las obtenidas en dos sitios de Jojutla, Mor. (13 especies, Deloya et al. 1987) y a las encontradas en Tecmilco, Tepoztlán, Mor. (14 especies, Deloya 1996). Estos sitios también albergan bosque tropical caducifolio y subcaducifolio. De este modo, nuestros resultados se encuentran dentro de los rangos de riqueza de especies para los bosques tropicales mexicanos, que según Deloya $\&$ Morón (1998) son de 8 a 11 géneros y 12 a 21 especies para caducifolios, y 9 géneros y de 14 a 22 especies para bosques subperennifolios. Del mismo modo los períodos de colecta restringidos a la época lluviosa se corresponden con la actividad de los Scarabaeidae de regiones tropicales y subtropicales, por lo cual se observa que la mayoría de las especies mostraron una mayor abundancia durante esa época y fue disminuyendo al avanzar hacia fines del otoño. La tribu Scarabaeini redujo su abundancia de Junio a Noviembre, Eurysternini solo apareció activo en Junio (mes 
con mayor precipitación y temperatura), algunos Coprini se comportaron igual que los Scarabaeini mientras que otros solo estuvieron activos durante Junio. Phanaeini a excepción de $C$. pluto estuvieron activos solo durante verano, Onthophagini presentaron una actividad diversa por especie, algunos se mantuvieron activos durante todo el estudio como los Scarabaeini y Coprini, mientras otros solo aparecieron en los periodos con mayor precipitación.

Las condiciones de suelos someros de tipo calizo, parecen favorecer el predominio en abundancia de las especies rodadoras, de los géneros Deltochilum y Canthon. Por el contrario las especies cavadoras, si bien cuantitativamente no están bien representadas por la necesidad de suelos profundos, superan en riqueza a las primeras, al ser favorecidas por la pérdida y deterioro de la vegetación natural. En la Reserva de la Biósfera de Sian Ka'an en Quintana Roo, que presenta suelos similares, Morón et al. (1987) señalaron la misma situación.

En muestreos utilizando trampas pit-fall con excremento vacuno como cebo, realizados en los mismos sitios se han colectado 25 especies de Scarabaeinae (EMO datos no publicados) de las cuales 14 (56\%) son comunes a este trabajo. Las once especies "faltantes" son consideradas estrictamente coprófagas por lo cual no responden a las trampas NTP. Entre ellas se encuentran especies de los géneros Dichotomius, Canthidium, Copris, Pedaridium, Canthon, Pseudocanthon, Onthophagus, y dos especies no nativas, Euoniticellus intermedius Reiche y Digitonthophagus gazella F. (EMO datos no publicados). Además de ellas, otras especies que no se registraron en nuestro trabajo están Canthon (Glaphyrocanthon) moroni Rivera-Cervantes \& Halffter, especie aparentemente necrófaga muy rara conocida solo por un ejemplar macho de Jalcomulco (450m), y Canthon (Glaphyrocanthon) zuninoi Rivera-Cervantes \& Halftter, especie copro-necrófaga de interior de bosque, conocida solo de Jalcomulco (450m) (Rivera-Cervantes \& Halffter 1999). Por su parte Canthon (Glaphyrocanthon) leechi coexiste con C. (G.) zuninoi y puede ser confundido con éste, a pesar de ser de zonas desmontadas y de borde de bosque. Sin embargo, ninguno de los ejemplares analizados pertenecientes al subgénero Glaphyrocanthon mostraron las características determinantes de $C$. (G.) zuninoi (élitros y pronoto brillantes, quilla supraclipeal continua, borde lateral del pronoto próximo al ángulo medio, ligeramente cóncavo, borde de la proepisterna con reborde y machos sin tuberculito entre los trocánteres III); caso contrario para $C$. (G.) leechi.

Entre los patrones de distribución en los insectos propuestos por Halffter (1976, 1978) para la Zona de Transición Mexicana, el Neotropical Típico (PNT) es el mejor representado con 12 especies. Dentro de este patrón están las especies de Uroxys con penetración mínima, Coprophanaeus, Deltochilum y Eurysternus con penetración media, y Phanaeus, Canthon, Dichotomius con penetración máxima, probablemente Ataenius también se encuentre en este patrón por distribución tropical y subtropical. 
El Patrón Paleoamericano está representado por especies de Onthophagus, Sysiphus y probablemente Omorgus por su distribución neártica con penetración al neotrópico.

Las afinidades de las regiones al parecer coinciden con el origen y composición de las faunas, ubicación geográfica de los sitios y distribución de especies. Apazapan-Jalcomulco, Palma Sola, El Cielo y Laguna Verde son regiones ubicadas en la Provincia Biogeográfica Golfo de México (sensu Morrone, 2005) y están compuestas principalmente por especies de origen neotropical. $D$. scabriusculum, $C$. pluto, C. leechi, O. batesi, D. amplicollis, $U$. deavilai, y las especies exclusivas de la vertiente atlántica $E$. mexicanus y $S$. mexicanus fueron registradas para todas las regiones de este grupo, separándolas de las otras y aumentando los porcentajes de similitud. Dentro de este primer grupo de regiones, los escarabajos copro-necrófagos C. telamon, O. schaefferi, P. endymion, U. deavilai, A. cribrithorax y las dos especies de Omorgus están registrados en una o mas de ellas como coprófagos, motivo por el cual los porcentajes de similitud no fueron mayores. La falta de información publicada de registros de la familia Trogidae y la tribu Euparini en algunos sitios fue otro factor que pudo haber elevado los porcentajes. Con base a los resultados obtenidos podemos considerar a esta región central baja de Apazapan-Jalcomulco y a Palma Sola como una misma unidad, lo cual coincide con lo mostrado por Halffter et al. (1995). Resulta interesante señalar las similitudes encontradas entre la región aquí estudiada con la región costera de Veracruz así como con la parte baja de la región de la Reserva de la Biósfera El Cielo en Tamaulipas, a pesar de su aparente aislamiento con ambas regiones en relación a su ubicación geográfica.

Por el contrario las regiones restantes, a excepción de los Tuxtlas, están conformadas por faunas de filiación múltiple, compuestas por un conjunto de especies de origen Neotropical, del Altiplano Mexicano y el Eje Volcánico Transmexicano. Estas regiones pertenecen a las Provincias Biogeográficas Costa Pacífica Mexicana (Puerto Ángel) y Cuenca del Balsas (Chamela, Jojutla, Acahuizotla) (sensu Morrone, 2005) y se caracterizaron por presentar especies distribuidas por la vertiente pacífica hacia el sureste como Pseudocanthon perplexus, Ataechus rodriguezi y Agamopus lampros.

Los Tuxtlas se ubica dentro de la Provincia Golfo de México, pero presenta bosque tropical perennifolio con elementos de origen sudamericano con penetración reciente y exclusivos de este tipo de vegetación como Deltochilum pseudoparile, Anaides laticolis, Ataechus illaesum y Bdelyropsis newtoni.

La existencia de sólo 23 géneros de Scarabaeidae y 2 de Trogidae para el país, hace que la información biogeográfica acerca de las diferencias entre regiones sean poco marcadas. Esto se debió a la combinación de géneros con orígenes, afinidad y patrones de dispersión comunes, encontrados en el centro del país, y representados por especies distribuidas en diferentes provincias y regiones del país. A pesar de esto se generaron dos grupos, uno muy grande en el que se agregaron las regiones 
Neotropicales, incluyendo una considerada como Neártica por su ubicación geográfica (Acahuizotla). El segundo grupo se conformó por regiones Neárticas (Chamela y Jojutla).

Este conjunto de características representan y aclaran las similitudes y afinidades de la composición de especies en las regiones desde una base histórica, biogeográfica y ecológica, a tal grado que algunos autores como Morrone (2001) han considerado a los patrones de dispersión y la configuración de las provincias biogeográficas como una homología geográfica.

El área de estudio mantiene una fauna de escarabajos necrófagos de amplia distribución y afinidades tropicales y templadas, aún al tener como matriz plantaciones de mango con parches de cultivos de maíz, pastizales y acahuales de bosque, mantiene. Este paisaje heterogéneo y modificado está imitando algunas condiciones ambientales de los bosques tropicales caducifolios y/o subperennifolios que le permiten soportar una comunidad de 11 géneros y 18 especies. Estos valores están ubicados en el rango expresado por Deloya y Morón (1998) para estos tipos de ambientes boscosos, por lo que podemos decir que estos ambientes están manteniendo una comunidad de coleópteros necrófagos similar al bosque tropical caducifolio en riqueza específica, ya sea funcionando como sitios de alimentación, como corredores entre los manchones de bosque o como refugio alternativo donde encuentran disponibilidad de recurso. Así mismo la presencia de otros restos y productos de materia orgánica derivados de la cada vez más creciente presencia y actividad humana propiciarían la disponibilidad de ciertos recursos utilizados por este grupo de escarabajos para su sobrevivencia. Sin embargo, la presencia de una abundancia mayor de escarabajos con hábitos nocturnos sugiere, que a pesar de que mantienen los rangos de riqueza especifica de los bosques, su composición se está modificando a tal grado que parecen ser de ambientes abiertos, modificados y/o perturbados. Es necesario encaminar esfuerzos a estudios enfocados al análisis de los cambios en el ensamble de las comunidades, vacantes de nichos y al efecto del cambio de uso de suelo sobre las especies con fines de conservación y manejo.

AGRADECIMIENTOS. El desarrollo de este trabajo fue posible gracias al apoyo y facilidades del Departamento de Biodiversidad y Ecología Animal del Instituto de Ecología AC.

\section{LITERATURA CITADA}

Arnaud, P. 2002. Les Coléopterès du Monde. Phanaeini. Dendropaemon, Tetramereia, Homalotarsus, Megatharsis, Diabroctis, Coprophanaeus, Oxysternon, Phanaeus, Sulcophanaeus. Hillside Books, Canterbury, United Kingdom.

Arellano, L. \& G. Halffter. 2003. Gamma Diversity: derived from and a determinant of alpha diversity and beta diversity. An analysis of three tropical landscapes. Acta Zoológica Mexicana (n.s.), 90: $27-76$. 
Colwell, R.K. 2005. EstimateSWin700. Statistical estimation of species richness and shared species from samples, Versión 7. Department of Ecology abd Evolutionary Biology, University of Connecticut, U.S.A. http://viceroy.eeb.uconn.edu/estimates.

Colwell, R.K. \& J.A. Coddington. 1994. Estimating terrestrial biodiversity through extrapolation. Philosophical Transactions of the Royal Society (Series B), 345:101-118.

Delgado, L. 1989. Fauna de Coleópteros Lamelicornios de Acahuizotla, Guerrero, México. Tesis de Licenciatura. UNAM, México, D.F. 154 pp.

Delgado, L. \& E. Montes de Oca. 2005. Los escarabajos copro-necrófagos. Pp. 405-416. En: SánchezRamos, G., P. Reyes-Castillo \& R. Dirzo (eds.). Historia Natural de la Reserva de la Biosfera El Cielo, Tamaulipas, México. Universidad Autónoma de Tamaulipas, México.

Delgado, L. \& B. Kohlmann. 2007. Revisión de las especies del género Uroxys Westwood de México y Guatemala (Coleoptera: Scarabaeidae: Scarabaeinae). Folia Entomológica Mexicana, 46 (1): 136.

Deloya, C. 1996. Los macro-coleópteros necrófilos de Tepoztlán, Morelos, México (Scarabaeidae, Trogidae, Silphidae). Folia Entomológica Mexicana, 97: 39-54.

Deloya, C. 2005. Omorgus rodriguezae especie nueva de México y clave para separar las especies del género para centro y norteamérica (Coleoptera: Trogidae). Folia Entomológica Mexicana, 44(Supl. 1): 121-129.

Deloya, C. \& M.A. Morón. 1994. Coleopteros lamelicornios del distrito de Jojutla, Morelos, México (Melolonthidae, Scarabaeidae, Trogidae y Passalidae). Listados Faunísticos de México. UNAM, V: $1-49$.

Deloya, C. \& M.A. Morón. 1998. Scarabaeoidea (Insecta: Coleoptera) necrófagos de "Los Tuxtlas", Veracruz y Puerto Ángel, Oaxaca, México. Dugesiana, 5(2): 17-28.

Deloya, C., G. Ruíz-Lizárraga \& M.A. Morón. 1987. Análisis de la entomofauna necrófila en la región de Jojutla, Morelos, México. Folia Entomológica Mexicana, 73: 151-171.

Deloya, C., V. Parra-Rabla \& H. Delfín-González. 2007. Fauna de los Coleópteros Scarabaeidae Laparosticti y Trogidae (Coleptera: Scarabaeoidaea) asociados al Bosque Mesófilo de Montaña, cafetales bajo sombra y comunidades derivadas en el centro de Veracruz, México. Neotropical Entomology, 36(1): 5-21.

Edmonds, W.D. 1994. Revision of Phanaeus MacLeay, a New World genus of Scarabaeinae dung beetles (Coleoptera: Scarabaeidae, Scarabaeinae). Natural History Museum of Los Angeles County, Contributions in Science, 443: 1-105.

Halffter, G. 1976. Distribución de los insectos en la Zona de Transición Mexicana. Relaciones con la entomofauna de Norteamérica. Folia Entomológica Mexicana, 35: 1-64.

Halffter, G. 1978. Nuevo patrón de dispersión en la Zona de la Transición Mexicana: el Mesoamericano de Montaña. Folia Entomológica Mexicana, 30-40: 219-222.

Halffter, G. \& W.D. Edmonds. 1982. The nesting behavior of dung beetles (Scarabaeinae). An ecological and evolutive approach. Instituto de Ecología, A. C., México, D. F.

Halffter, G., M.E. Favila \& V, Halffter. 1992. A comparative study of the structure of the scarab guild in Mexican tropical rain forests and derived ecosystems. Folia Entomológica Mexicana, 84: 131156.

Halffter, G., M.E. Favila \& L. Arellano. 1995. Spatial distribution of three groups of Coleoptera along an altitudinal transect in the Mexican transition Zone and its biogeographical implications. Elytron, 9: $151-185$.

Howden, H. 1966. Notes on Canthonini of the "Biología Centrali-Americana" and descriptions of new species (Coleoptera: Scarabaeidae). Canadian Entomologist, 98: 725-741. 
Howden, H. \& O. Cartwright. 1963. Scarab beetles of the genus Onthophagus Latreille north of Mexico (Coleoptera-Scarabaeidae). Proceedings of the U. S. National Museum, 114 (3467) 133 pp.

Jessop, L. 1985. An identification guide to Eurysternine dung beetles (Coleoptera: Scarabaeidae). Journal of Natural History, 19: 1087-1111.

Jiménez-Valverde, A. \& J. Hortal. 2003. Las curvas de acumulación de especies y la necesidad de evaluar la calidad de los inventarios biológicos. Revista Ibérica de Aracnología, 8: 151-161.

León-Cortés, J.L., J. Soberón-Mainero \& J. Llorente-Bousquets. 1998. Assessing completeness of Mexican sphinx moth inventories through species accumulation functions. Diversity Distribution, 4: 47-44.

Kohlmann, B. \& A, Solís. 2001. El género Onthophagus (Coleoptera:Scarabaeidae) en Costa Rica. Giornale Italiano di Entomologia, 49(9): 159-261.

Montes de Oca, E. \& G. Halffter. 1995. Daily and seasonal activities of a guild of the coprophagous, burrowing beetle (Coleoptera, Scarabaeidae, Scarabaeinae) in tropical grassland. Tropical Zoology, 8: $159-180$.

Morón, M.A. 1979. Fauna de los Coleópteros lamellicornios de la Estacion de Biología Tropical "Los Tuxtlas", Veracruz, UNAM, México. Annales del Instituto de Biologia de la UNAM. Serie Zoología, 50(1): 375-454.

Morón, M.A. 1996. Cap. 21. Scarabaeidae (Coleoptera). Pp. 309-328. En: Llorente-Bousquets, J., A.N. García A. y E. González S. (eds.). Biodiversidad, taxonomía y biogeografía de artrópodos de México: Hacia una síntesis de su conocimiento. CONABIO-IB-UNAM, México, D.F.

Morón, M.A. (Ed). 2003. Atlas de los escarabajos de México. Coleoptera: Lamellicornia. Vol. II Familias Scarabaeidae, Trogidae, Passalidae y Lucanidae. Argania Editio. Barcelona, España.

Morón, M.A. \& R. Terrón. 1984. Distribución altitudinal y estacional de los insectos necrófilos de la Sierra Norte de Hidalgo, México. Acta Zoológica Mexicana (n.s.), 3:1-47.

Morón, M.A., J.F. Camal \& O. Canul. 1987. Análisis de la entomofauna necrófila del área Norte de la Reserva de la Biósfera "Sian Ka' an”, Quintana Roo, Mexico. Folia Entomológica Mexicana, 69: 83-98.

Morón, M.A., C. Deloya \& L. Delgado.1989. Fauna de coleópteros Melolonthidae, Scarabaeidae y Trogidae de la región de Chamela, Jalisco, México. Folia Entomológica Mexicana, 77: 83-98.

Morrone, J.J. 2005. Síntesis biogeográfica de México. Revista Mexicana de Biodiversidad, 76(2): 201252.

Morrone, J.J. 2001. Halffter's Mexican Transition Zone, beetle generalized traces, geographical homology. Journal of Biogeography, 28: 635-650.

Rivera-Cervantes, L. \& G. Halffter. 1999. Monografia de las especies mexicanas de Canthon del subgénero Glaphyrocanthon (Coleoptera: Scarabaeidae: Scarabaeinae). Acta Zoológica Mexicana (n.s.), 77: 23-150.

Rzedowski, J. 1986. Vegetación de México. Edit. Limusa, México, D.F., $3^{\text {a }}$ reimp., 432 pp.

Soberón-Mainero, J. \& J. Llorente-Bousquets. 1993. The use of species accumulation functions for the prediction of species richness. Conservation Biology, 7(3): 480-488.

Statsoft. 1999. Statistica 5.5 for Windows. StatSoft Inc. Oklahoma, USA. 
Mora-Aguilar y Montes de Oca: Escarabajos necrófagos del centro de Veracruz

\section{Apéndice}

Clave para determinar las especies de escarabajos obtenidos en NTP-80 en Apazapan y Jalcomulco

1 Canto ocular ausente. Cavidades mesocoxales cerradas lateralmente, separadas de los mesepímeros. Abdomen con cinco esternitos visibles. Placa pigidial siempre oculta por

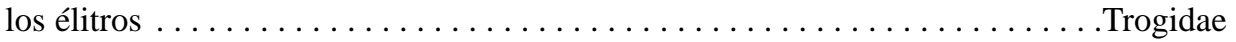
Antenas con el escapo alargado y el pedicelo unido por debajo del ápice del escapo. Escutelo astado, constriñendose en la base .................. Omorgus ...2

1' Cara interna del profémur sin surco en la mitad anterior para recibir a la protibia. Cavidades mesocoxales abiertas lateralmente, en contacto con los mesepímeros. Abdomen con seis esternitos visibles. Placa pigidial expuesta o parcialmente oculta por

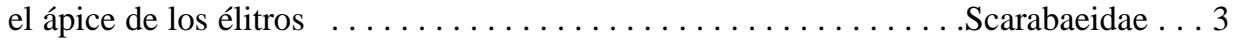

2 Pronoto débilmente convexo, al menos anteriormente, débilmente deprimido o tuberculado excepto ligeramente en la base; élitros débilmente costillados. Lados del pronoto agudamente angulados en su tercio basal y con sedas finas, largas próximas entre sí .................................. suberosus Fabricius, 1775

$2^{\prime}$ Pronoto con carenas, tubérculos y depresiones profundas; costillas elitrales tuberculadas tomentosas y opacas. Edeago con los lóbulos laterales su mitad apical ligeramente paralelos, ápice redondeado . . . . . . . . . . . . . . . . fuliginosus Robinson, 1941

3 Metatibias con dos espolones (Aphodiinae). Meso y metatibias sin carinas transversales (Euparini). Pigidio escabroso sin gránulos. Ángulo posterior del pronoto no truncado con puntuación irregular en las dos terceras partes anteriores. Superficie lateral del pronoto con puntuación heterogénea .................Ataenius cribritorax Bates, 1887

3' Pigidio expuesto completamente. Metatibias con un espolón apical .Scarabaeinae . . . 4

4 Meso y metatibias alargadas y esbeltas, escasas y gradualmente ensanchadas hacia el ápice. Cabeza y pronoto careciendo de cuernos y protuberancias . . . . . . . . . . . 5

4' Meso y metatibias cortas y robustas, fuertemente y en ocasiones abruptamente ensanchadas apicalmente. Cabeza y pronoto generalmente con cuernos y protuberancias $\ldots \ldots \ldots \ldots .7$

5 Élitros usualmente redondeados y curvados gradualmente hacia los lados. Palpos labiales con tres artejos . . . . . . . . . . . . . . 6

5' Élitros aplanados dorsalmente y doblados angularmente hacia los lados. Palpos labiales con dos artejos (Eurysternini). Pronoto con tres manchas brillantes sin sedas en la parte media anterior. Metafémures sin proyecciones en el margen posterior . ............

.Eurysternus mexicanus Harold, 1869

6 Pronoto no comprimido, con los lados doblados oblicuamente hacia abajo. Elitros redondeados desde la base al ápice. Metatarsos poco largos, y más o menos comprimidos

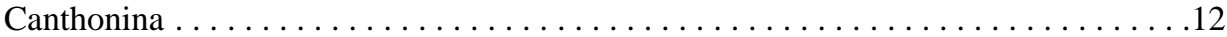

6' Pronoto comprimido lateralmente, con los lados verticales. Élitros convergentes desde su base al ápice. Metatarsos muy largos, gráciles y filiformes (Sisyphina). Punteado pronotal amplio y poco profundo. Borde inferior de las metatibias con una hilera de 9-11 dentículos redondeados ..................Sisyphus mexicanus Harold, 1863 
7 Tercer artejo de los palpos labiales inconspicuo, reducido o ausente. Escutelo no visible o inconspicuo. Antenas con nueve artejos (Onthophagini) . . . . . Onthophagus ...... . 8

$7^{\prime}$ Tercer artejo de los palpos labiales bien desarrollado . . . . . . . . . . . . . 10

8 Superficie del pronoto sin sedas. élitros con sedas en la epipleura. Machos con un par de cuernos en la base de la cabeza y unidos por una carina fina. Puntuación marcada entre las coxas del metaesternón ............... O. batesi Howden \& Cartwright, 1963

8' Superficie del pronoto y élitros cubierta por sedas evidentes, cortas o largas. Machos sin clípeo proyectado. Puntuación pronotal simple, reborde redondeado. Élitros o cuerpo pueden ser bicolor. Tamaño pequeño, $4.2-5.1 \mathrm{~mm} \ldots \ldots \ldots \ldots \ldots \ldots \ldots$

9 Base del pronoto completamente marginada; élitros bicolor, normalmente con áreas amarillas irregulares hacia su base y ápice y por lo menos con algunas manchas redondas amarillas pequeñas en el disco. Machos con mejillas poco extendidas y protibias finas con el cuarto diente poco desarrollado o ausente .......... . höpfneri Harold, 1869

9' Base del pronoto finamente marginada en su parte media; élitros unicolor, oscuros o claros, o si bicolor, formado por bandas oscuras en algunas interestrías. Machos con mejilla muy extendida, protibias gruesas y con el cuarto diente desarrollado ......... .............................. schaefferi Howden \& Cartwright, 1963

10 Artejo basal de la maza antenal normal, no excavado. Protarsos presentes y con uñas. (Coprini). Cada élitro con siete u ocho estrias discales y en ocasiones con una más sobre la epipleura. Surco pronotal medio longitudinal ausente o débilmente impreso .......

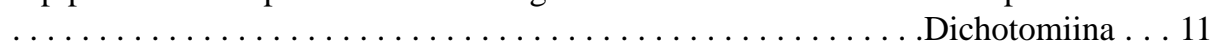

10` Artejo basal de la maza antenal excavado para recibir los artejos distales. Protarsos ausentes, o si están presentes carecen de uñas . . . . . . . . . Onitini, Phanaeina . . 15

11 Metatarsos con el artejo basal triangular. Clípeo rugoso. Cabeza con tubérculos o cuernos y pronoto generalmente con quillas o tubérculos. Macho con el área fronto-clipeal con una protuberancia trituberculada, la central más elevada, truncada y excavada con el ápice ......................... Dichotomius amplicollis (Harold, 1869)

11' Surco basal del pigidio moderadamente trisinuado. Área dorsal ocular cuatro veces más larga que ancha; distancia interocular ocho veces el ancho dorsal de un ojo. Élitros ovados; machos con el ápice de los élitros moderadamente proyectado sin dientes .... ........................... Uroxys deavilai Delgado \& Kohlmann 2007

12 Élitros con el borde lateral marcado por una fuerte quilla. Ápice de las interestrías elitrales con quillas o tubérculos. Protarsos ausentes .......... Deltochilum . . 13

$12^{\prime}$ Élitros con el borde lateral sin quilla. Ápice de las interestrías elitrales sin quillas o tubérculos ..................................... 14

13 Élitros toscamente rugosos, con zonas brillantes sin puntos; clípeo bidentado. Cada élitro con una quilla humeral que se extiende hasta cerca del callus apical. Quilla epipleural bien marcada pero poco prominente ....D. scabriusculum scabriusculum Bates, 1887

13' Élitros poco rugosos, con una quilla humeral que se extiende un poco mas allá del nivel de articulación de las metacoxas, junto a su base hay un tubérculo alargado. Clípeo cuatridentado. Machos con un par de gibas elitrales y las metatibias anguladas ..... ..D. gibbosum subleave Bates, 1887 
14 Cara ventral del fémur posterior no marginada anteriormente. Borde lateral de la proepisterna sin reborde o dentículo en su margen anterior. Clípeo bidentado. Superficie dorsal con chagrinado evidente. Machos con un pequeño tubérculo entre los trocánteres II ........................... leechi (Martínez, Halffter \& Halffter, 1964)

14' Cara ventral del fémur posterior con una fina quilla anterior. Fémur anterior no dentado en los machos. Metafémures con el borde anterior finamente marginado. Clípeo cuatridentado ..................... cyanellus cyanellus LeConte 1859

15 Ápice del clípeo con tres escotaduras profundas delimitando dos dientes agudos centrales

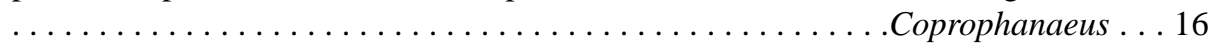

15 Ápice del clípeo con una escotadura central muy ligera u obsoleta y sin dientes conspicuos agudos. Pronoto con el borde lateral recto o redondeado detrás del ángulo anterior. Margen pronotal anterior roto detrás de los ojos . . . . . . . Phanaeus . . 17

16 Machos con una proyección laminar sinuada en la frente y dos protuberancias en el pronoto. Hembras con una carina trituberculada frontal y una quilla transversal redondeada en el pronoto .................. telamon corythus (Harold, 1863)

16' Machos con una quilla frontal tridentada y una proyección voluminosa ampliamente bifurcada en el pronoto. Hembras con una carina frontal trituberculada y una quilla transversal ligeramente hendida en el pronoto ............. . pluto (Harold, 1863) Pronoto densamente granulado anterolateralmente. Interestrías con puntos diminutos. Disco pronotal en machos bien desarollados concavo anteriormente con bordes lateralmente levantados. Proceso medio posterior usualmente elongado, con ápice bifurcado ................................... tridens Laporte, 1840

17' Pronoto casi liso débilmente punteado anterolateralmente. Interestrías convexas y brillantes, estrias con fosas en la base. Machos con pronoto triangular, disco pronotal con el centro liso y lateralmente áspero, ángulos posteriores dirijidos lateralmente. . ..... 